\title{
el PREMATURo NACIDO EN EL hospitAL CeINICO REGIONAL EN 1954
}

\author{
DrS. DANIEL CAMPOS y CARLOS TREUER \\ Servicio de Pediatria. Jefe: Dr. Daniel Campos. \\ Hospital Clínico Regional. Concepción.
}

Frente a la preocupación que hoy existe por el estudio del prematuro, resulta imposible $\mathrm{y}$ aún culpable permanecer indiferente. Ya era tiempo de analizar este problema en nuestro medio hospitalario y tratar de ver qué sucede con él, hasta donde nuestras posibilidades lo permitan.

Ante todo, debemos exponer los recursos $\mathrm{y}$ forma de atención para comprender mejor las limitaciones de este trabajo.

La asistencía del prematuro comienza intnediatamente que ha nacido. Ya en la sala de patto es atendido por el pediatra de guardia. Si pesa mas de 2.200 grs. y no ofrece nada de especial, e] niño pasa al lado de su madre en la sala de la Marernidad. Cuando media coliermedad o incervención de la madre, va a ba Sala Cuna. Si pesa menos de 2.200, complicado o no. se le descarga directamente a la Sala Incabadora y alli recibe la atcnción que detallatemos. Como no posemos incubadora de ningún tipo. sc procura acondicionar la sala a una temperatuta de 22 grados y se comptementa en la euna. con calentadores de diversos lipos. La hunedad varia según las condiciones ambicntales. Aqui se tecibe exclusivamente a aquellos que nacj. dos en nuestra Maternidad, son llevados de inmediato a la Sala Incubadora: se excluyen y se destinan a salas corrienres de Lactantes a los que ocasionalmence han permanecido algún tímpo en sala cana 0 con su madte $y^{r}$ a los que virnen de fuera. Se dispone de seis cunas. separadas por tabiques de vidrio, pero generalmente el número de prematuros alcanza a diez o doce, lo que. como se comprende, favorece la infección 4:3. La sala forma parte de la Sección de Lactanres del Servicio de Pediatria y está situada frente a la cocina de leche. La asistencia de cllos está a cargo de un pediatra de tres horas. que es al mismo tiempo Jefe de la Sección Lactantes (32-50 lactantes y 30.50 R. N.) y durante el resto del dia, a cargo del pediatra de guardia. Hay una entermera con seis horas para toda la sección $y$ cuatro aluxiliares enrrenadas. que bacen turnos rotativos; de modo que durante el dia (de 8 a 20 horas), siempre hay dos. y und en la noche.

Se procura mantener la téenica aséptica, augque ré sulta a reces muy dificil por diversos factores como son: la falta de ropa, la asistencia de alnmnos o cambios de personal. Eventualmente se usa luz ultravioleta desde tubos colocados en el cielo raso. Ingresado el niño. se le viste con ropa caliente. esterilizada y se le coloca en cuna, con calentadores. Protrción desde el presente año, con penicilina $100.000 \mathrm{U}$. por $\mathrm{kg}$. de peso-dia, estreptomicina 40 mgrs. por kg. pesodi $2 y$ vitamina $K 5$ mgrs, cada $12-24$ horas dutante siete días $2 ! !$.

Se les administra oxígeno humidificado cuando bay necesidad. Si el estado del prematuro lo exige, se le deja en el resucitador hasta que su recuperación total o parcial permita transferirlo a la sala. Vale decir. cuando se ha hecho la aspiración de secreciones y ha mejorado la oxigenación.

Alimentución: nada por boca durante 24-48 horas según ef estado del niño y luego Eledón al $10 \%$ con $5 \%$ di maltosa-dextrina con sonda de goma. gotario - biberón. Se administran 10 tomas al dia cada dos horas, comenzando por $\mathbf{5}$ grs. cada vez, y aumentindo según la colerancia hasta llegar a una dosis ajustada a los requerimientos calóricos. No usamos sonda de potietileno por falca de recursos para adquirirla. Tampoco empleamos la leche humana por la dificultad pata obtenerla ya que las madres son dadas de alra precón- 
mente y hay poco personal paci su recolectión. Agua segrin necesidad. Como agregado vilamínico se las da 1.500 U. de vitamina D y 25 mgrs. de vitamina $C$ deste la segunda semanz.

Se lliva una cursa de peso y de temperatura rectal cn yue ademis se dnoti la alimentación y tracamiento.

Lu flebacisis y transiusión de sanger o de plasma no las usamos practicamente por fakla de personat adiesriado. ni zampoco las otras clisis por el rissgo que pueden envolver. salvo casos bien calificados.

Contamos con la colaborasion de los laboratorios centrales del Hospital e Institutos universitarios. El Servicio Social coopera con encueslas y colocacioncs. Alt $\mathrm{rot} 2.500 \mathrm{grs}$. previa la vacunacion BCG $\mathrm{s}$ instruccion de la madri.

Como st pucde aprecias. nucstra atención dei premaluto difiere muy poco di la que se hacía hace catotce años en algunos hospitales de la capital $1 \times$ y dista muchisimo de lo que detualmente se hace en lus principales clinicas pediátricas, tanco del extranje-

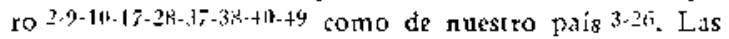
modalidades de atención las podemos apreciar a traves

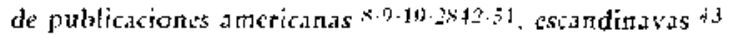
$y$ nucionales $\mathbf{i} \cdot 2 \pi$.

\section{MATERIAL}

Analizaremos en seguida el material de prematuros atendido durante el año 1954 en el Hospital Clínico Regional:

Estudiando el cuadro $\mathrm{N}^{\circ} 1$, tenemos:

Nacidos vivos: 3.857 .

Prematuros: nacidos vivos $248(6,42 \%$ $\sin$ significado estadístico).

Mortalidad total de recién nacidos: 190 (4,9\% sobre el total de nacidos vivos).

Mortalidad global de prematuros: $20,56 \%$ sobre el total de prematuros.

De los 248 hubimos de eliminar 19 observaciones que estaban incompletas por diferentes motivos y revisamos las 229 restantes con las respectivas historias de sus madres, lo que da un total de 458 fichas estudíadas. De estos 19 , fallecieron 5 . He-
CLALRO $\mathrm{S}^{\circ} 1$

MORTALIDAD EN RECIEN NACIDOS

\begin{tabular}{|c|c|c|c|c|c|c|c|c|}
\hline \multirow[b]{2}{*}{ R. naะidos } & \multicolumn{4}{|l|}{1} & 1 & \multicolumn{3}{|c|}{ Mortalidad } \\
\hline & $\mathrm{i}$ & $\mathbf{N} !$ & & $\%$ & 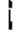 & $\mathbb{N}:$ & $\vdots$ & $\%$ \\
\hline f'rentaltures - & 1 & 248 & 1 & 6,72 & i & 51 & I & 20.56 \\
\hline 1) : & I & $\left.3 . v^{4}\right)$ & I & $9.7,58$ & | & 139 & & 3,9 \\
\hline 'TCO'AC. & 1 & 3.857 & 1 & $10 !$ & 1 & 194 & i & 4,9 \\
\hline
\end{tabular}

chas las correcciones nos quedan 46 fallecidos $(20,08 \%)$.

En el cuadro No 2, se relaciona la mortalidad de nuestra casuística con el peso y se la compara con otras estadisticas 3.5. 25.38 52.

Se observa lo comunicado por todos los autores 22.4 , tal es, que a mayor peso menor mortalidad; puesto que para los de menos de 1.000 grs. hay $100 \%$ y para los grupos sucesivos se nota disminución gradual siendo $4,62 \%$ en el grupo de mayores de 2.000 grs. Si reducimos nuestra muestra a los menores de $2.000 \mathrm{gr}$, la mortalidad alcana al $67,8 \%$, cifra extraordinariamente elevada en comparación con la del Chicago-Lying in Hospital "s que es de $39 \%$.

Todas las cifras de autores extranjeros dan tasas inferiores a las nuestras en cualesquiera de los grupos correspondientes, lo que es tanto más grave cuanto que nuestra estadistica abarca sólo un año. Los autores nacionales comunican en sus series cifras inferiores a las obtenidas por nosotros, en los que pesan menos de $2.000 \mathrm{gr}$. no así en los de mayor peso.

También hemos comparado la mortaljdad en relación con el sexo, puesto que es conocida la mayor proporción de hombres fallecidos ${ }^{49}$. En nuestra serie nacieron más mujeres, 54.14 $\%$ y su mortalidad fué significativamente igual a la de los varones. Asimismo, la proporción de úni$\cos$ es notoriamente mayor $(83,4 \%)$ que

ClALRO

MORTALIDAD EN RELACION AL PESO DE NACIMIENTO

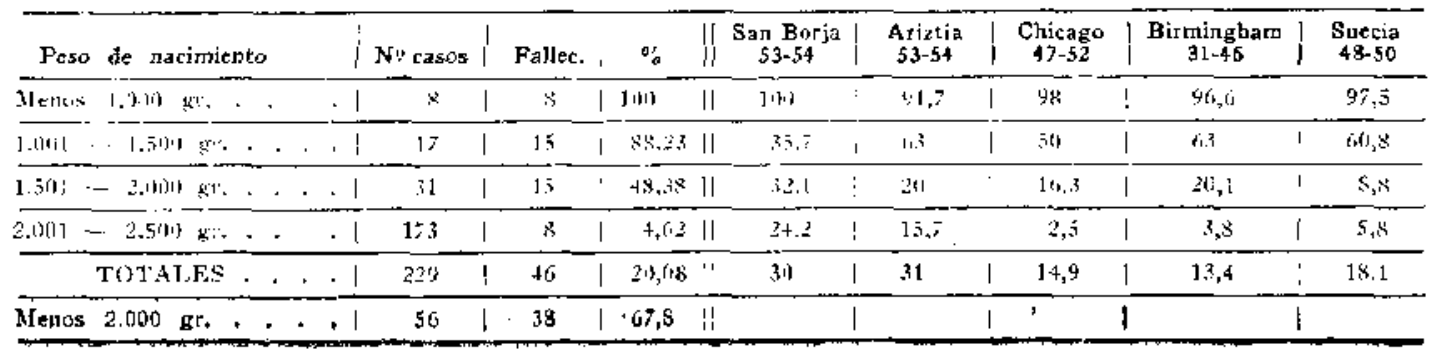




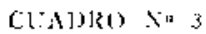

MORTALIDAD: CAUSAS $Y$ FRECUENCIA EN LA PRIMERA SEMANA

\begin{tabular}{|c|c|c|c|c|c|c|c|c|c|c|c|c|c|c|c|c|c|c|}
\hline Causas & & & I & $24 \mathrm{~h}$ & . & 48 & I & 72 & 1 & 96 & 1 & $6 D$. & i & $7 \mathrm{D}$ & I & $1 \mathrm{~S}$. & । & $\%$ \\
\hline He:no. lntrac. & , . & $\cdot$ & $\cdot 1$ & $\vdots$ & $\mathrm{i}$ & 7 & 1 & 3 & 1 & 1 & $\therefore$ & & i & 7 & 1 & 17 & $i$ & 4.3 .53 \\
\hline Posumaturishat. & $\cdot$. & & $\dot{.}$ & 7 & b & 1 & I & & $!$ & & I & 1 & 1 & & 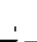 & 9 & 1 & 23,07 \\
\hline Atelectasia . & $\cdot$ & $\cdot$ & $+\vdots$ & 4 & I & & 1 & & 1 & & I & & 1 & & 1 & + & 1 & 10,25 \\
\hline Asiixia . . . & . & $\therefore$ & $\because$ & 2 & 1 & 1 & $i$ & & 1 & & 1 & & 1 & & 1 & 3 & 1 & 7,69 \\
\hline Sitiins conte. . : & . & . & 1 & 2 & 1 & & 1 & & $\therefore$ & & $!$ & & 1 & & $i$ & 2 & i & 5,13 \\
\hline Iitruriconesuntunia & $\cdot$. & . & .1 & & I & $I$ & . & & 1 & & & & 1 & & 1 & 1 & 1 & 2,56 \\
\hline Sepsis . . . & . & $\therefore$ & .1 & & 1 & 2 & & & 1 & & 1 & & 1 & & 1 & 2 & 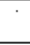 & 5.12 \\
\hline Malformaciones & $\therefore$ & - & $\cdot 1$ & 1 & 1 & & & & 1 & & . & & 1 & & 1 & $\mathbf{l}$ & 1 & 2,56 \\
\hline TOTSIES . & . . & + & .1 & 21 & 1 & 13 & I & i & I & 1 & I & J & I & ] & I & 39 & 1 & $94.5 \%$ \\
\hline
\end{tabular}

la de los gemelos $(16,59 \%)$ y respectivamente su mortalidad, $21,4 \%$ y $13,15 \%$.

En el cuadro No 3 se analizan las causas de mortalidad y su frecuencia en la primera semana. La mayor proporción de prematuros falleció en las primeras 24 horas (más de la mitad); en las próximas 24 horas fallecen los $2 / 3$ de los restantes. La causa de muerte más frecunete entre nosotros fué la hemorragia intracraneana, 43,$58 ; n$, 4.75 ; le sigue en proporción francamente decreciente la prematuridad propiamente tal, los trastornos respiratorios, la sifilis, infeeciones y malformaciones. Los trastornos respiratorios, sin embargo, ocupan el primer lugar en otras estadísticas 21-35.4?.

En el cuadro N 4, se resumen los porcentajes de mortalidad en la primera semana y en las restantes. Así, el $84,78 \%$ fallece en la primera semana de vida $\mathrm{y}$ solamente el $15,21 \%$ en las posteriores.

Comparando la mortalidad de Puericultura (sala cuna y salas en general) con la Sala Incubadora, tenemos que la mayor proporción de niños permaneció en Puericultura $(69,86 \%)$ y su mortalidad alcanzó a $6.25 \%$; en cambio, en Incubadora hubo $30,13 \%$ del total de prematuros $y$

$$
\text { CLATRO XI: } 4
$$

DISTRIBUCION DE LOS FALLECIDOS POR SEMANAS

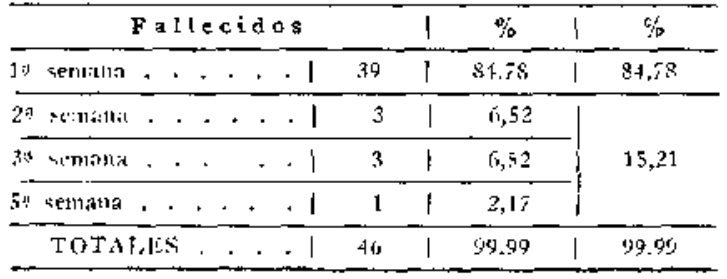

falleció el $52,17 \%$. El sobreviviente de menor peso fué de 1.400 gr.

Mortalidad y primi o multiparidad. Hay una notable mayor proporción de multíparas $(69,8 \%)$ y la mortalidad de sus hijos prematuros es menor $(18,75 \%)$ que la de los nacidos de primíparas $(23,1$ 's $)$.

Mortalidad de niños prematuros nacidos de madres sanas y de madres con alguna alteración patológica. Podemos decir que el 61,4 , tuvo una gestación normal y un $38,8 \%$ presentó alguna alteración significativa (toxemia, placenta previa, tuberculosis, hidroamnios, etc.). En el grupo de las madres sanas fallece el $16,4 \%$ de los niños; en cambio, en el otro hubo un $25,8^{\prime} \%$ de mortalidad.

Mortalidad en relación con el tipo de parto. Se eliminan 8 casos cuya muerte ocurrió tardiamente y no guardó relación con el parto. La mayor frecuencia corresponde a los nacidos de vértice $(81,44 ;, ;)$ con una mortalidad de $13,88 \%$, Ie sigue la presentación de nalgas $(12,66 \%)$ con una mortalidad de $42,85 \%$. EI fórceps se aplicó en un $3,61 \%$ de los casos y no hubo muertos. Finalmente la cesárea se practicó en un $2,26 \%$ de los casos y tuvo una mortalidad del $20 \%$.

En el cuadro $\mathrm{N}$, hemos relacionado la mortalidad con el estado civil de las madres y su asistencia prenatal. El $67,24 \%$ de las madres eran casadas y el $32,75 \%$ solteras. Por otro lado, la mayor proporción $(\mathbf{5 7}, \mathbf{5} \%)$ estuvo controlada en algún Servicio durante su embarazo. Se observa una notoria mayor mortalidad en el grupo de madres no controladas, como asimismo una mayor mortalidad en el de las solteras. 


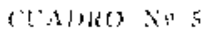

MORTALIDAD Y CONTROL DEL EMBARAZO

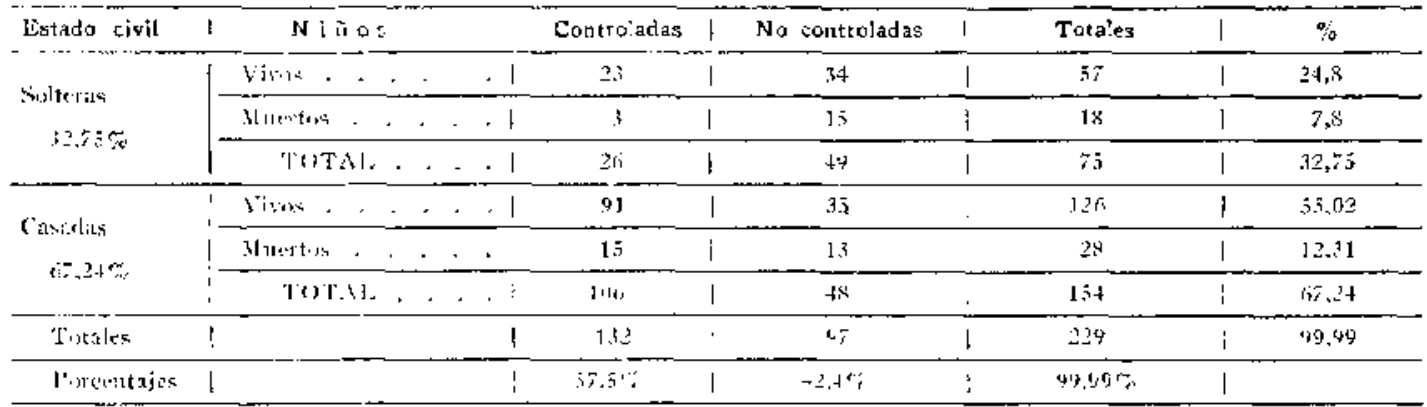

En el cuadro $N^{\circ} 6$, se presentan las causas de prematuridad de nuestro material. La mayor proporción corresponde a las causas desconocidas $(39,73 \%)$, seguida muy de cerca por las maternas $(37,5)$; luego, vienen las causas ovulares $(22,31 \%)$, habiendo sólo $0,4 \%$ de causas fetales.

En el cuadro N' 7 , se analizan las causas maternas de prematuridad, observándose que la toxemia parece ser, en nuestro medio, la principal causa materna: 32,$5 ;$ a continuación viene la primiparidad precoz $15 \%$, o sea, aquellas primíparas menores de 20 años len realidad todas estaban por debajo de los 18 años de edadi. Otro porcentaje importante Io da la multiparidad (gran multípara) con $10,46 \%$. La sifilis tuvo una proporción de $9,3 \%$ La prematuridad habitual $8,13 \%$ Esta es una denominación arbitraria con la que queremos englobar a aquellas madres cuyos hijos eran prematuros en su casi totalidad, sin que se divisara otra causa. La placenta previa, enfermedades infecciosas, nefritis, desprendimiento prematuro de placenta y otras, tuvieron escasa incidencia.

Entre las causas ovulares, predomina el enbarazo múltiple, gemelar en nuestro caso $(54.9 \%)$; en seguida viene la ruptura intempestiva de membranas $(37,25 \%) \mathrm{y}$ por último el hidroamnios $(7,84 \%)$.

CITITRO No G

CAUSAS DE PREMATURIDAD

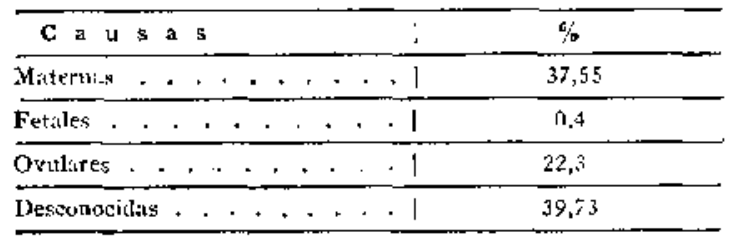

En las causas desconocidas (Cuadro N: 81 , exponemos solamente algunos de los factores que influirian, puesto que las anotaciones de las historias clínicas no estaban hechas intencionadamente, de modo que datos como las relaciones sexuales de los ultimos meses y salario, no los incluimos. Este último dato. sin embargo, lo consideramos, junto con vivienda, higiene, alimentación, etc., dentro de las condiciones generales, Observamos que el $7,69^{\prime} ;$ tenia buenas condiciones; $38,46 \%$ regulares y más de la mitad $(53,84 \%)$ malas.

En relación al estado civil había un $67,24 \%$ de madres casadas y un $32,75 \%$ de solteras. La convivencia la encontramos en un $79,12 \%$ y la profesión demostró que las tres cuartas partes $(75,83 \%)$ se dedicaban a labores de casa. Detallando, resulta: obreras 10,$98 ; ;$; domésticas $12,08 \%$ y profesoras $1,09 \%$.

Sabido es que la asfixia causa más de la mitad de las muertes en el período neonatal ", y que sus consecuencias ulteriores tienen mayor significación. Por ello estudiamos su frecuencia y sus probables causas en nuestro material (Cuadro No 9).

(C)

Calisas maternas de PREMaturidad

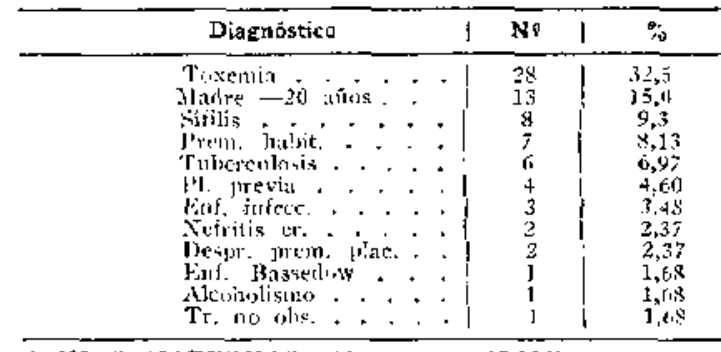

CACQAS MATERNAS: 66 C:LON $=37,55 \%$ 
CLABl: Xns

PREMATURIDAD DE CAUSA DESCONOCIDA

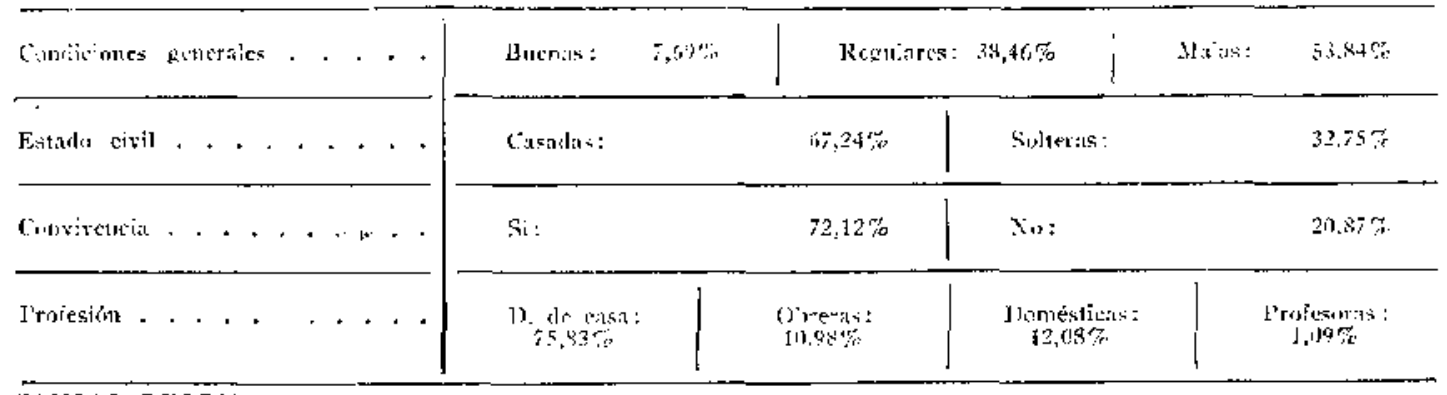

CALISAS DESCONOCIUAS: 91 casise = 39,73:

La frecuencia global fué de $23,1 \%$, con una mortalidad de $47,1 \%$. Sus causas fueron variadas, desde alteraciones del cordón a la inmadurez propiamente tal, considerando las alteraciones placentarias, malformaciones, drogas administradas a la madre y obstrucción de las vías aéreas. Naturalmente que la tolerancia a ella, fué notoriamente mejor en los prematuros de mayor peso, que en los pequeños donde fué mayor la mortalidad.

En el cuadro N? 10, estudiamos la frecuencia y tipos de infección $(36,68 \%)$. Garcés y col. ${ }^{12}$ dan $80,07 \%$ de infecciones con $26,87 \%$ de letalidad global. Entre nosotros, la onfalitis y la sepsis fueron las causas más frecuentes $(9.6 \%$ y $9 \%$, respectivamente). Su presencia fué reconocida entre los días, $2^{\circ}$ a $17^{\circ}$, con una mayor incidencia dentro de los 2 a 6 primeros dias de vida. La sepsis mostró una letalidad de un $10 \%$. Sigue en frecuencia la conjuntivitis uni o bilateral, con un porcentaje de $8,4 \%$. Las otras in- fecciones muestran escasa frecuencia $y$ fueron: sífilis congénita asintomática y sintomática ( $4 \%$ en conjunto), bronconeumonia, rinitis, faringitis, piuria y meningitis.

\section{COMENTARIO}

Aun cuando en si mismo el problema de la prematuridad tiene una escasa incidencia (5-10\%), cobra una enorme importancia al estudiar su influencia en la mortalidad infantil y especialmente neonatal. En ambos casos constituye una serio problema, ya que todas las estadísticas demuestran que un gran porcentaje de los fallecidos en estos dos rubros, son prematuros.

Ante la magnitud del problema nos propusimos estudiar lo que sucede en nuestro medio. Lo iniciamos tomando como muestra el material de 1954, sin desconocer que no podríamos deducir conclusiones, pero que, sí, nos revelaría ci-

CUADRO NO 9

CAUSAS DE ASFIXIA

\begin{tabular}{|c|c|c|c|c|c|c|c|c|c|c|c|c|}
\hline $\mathrm{C}$ a us a s & I & Menos 1000 & I & $1001-1500$ & I & $1501-2000$ & 1 & $2001-2500$ & 1 & Total & I & Fallecidos \\
\hline [1. previa, . . & .1 & & I & & $\vdots$ & & 1 & 2 & 1 & 2 & i & 1 \\
\hline ]). I's, Frlac. & .1 & & 1 & 1 & 1 & & I & 1 & 1 & 2 & 1 & 1 \\
\hline Cosistrín . . - & .1 & & I & & 1 & 2 & 1 & 5 & $!$ & 7 & 1 & 2 \\
\hline An. Scilanies . . & .1 & & 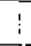 & 1 & 1 & 2 & 1 & 3 & 1 & G & I & 2 \\
\hline Ois. víls resple. & .1 & 1 & 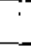 & 4 & 1 & 9 & I & 15 & I & 29 & $\mathrm{i}$ & 12 \\
\hline M[alianimats. : : & 1 & & 1 & & i & 1 & 1 & & 1 & 1 & 1 & 1 \\
\hline Inmurl:arez ... & .1 & $\therefore$ & 1 & & $!$ & & 1 & & 1 & 6 & 1 & " \\
\hline TOTAT. & $\therefore 1$ & 7 & 1 & 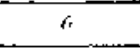 & $i$ & 14 & 1 & 26 & 1 & 5 & 1 & 25 \\
\hline Follecidera.... & .1 & $\overline{7}$ & i & 5 & 1 & 0 & 1 & $\hat{s}$ & 1 & 25 & i & \\
\hline
\end{tabular}




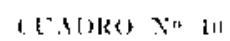

I $N$ F E C C C I O N E S

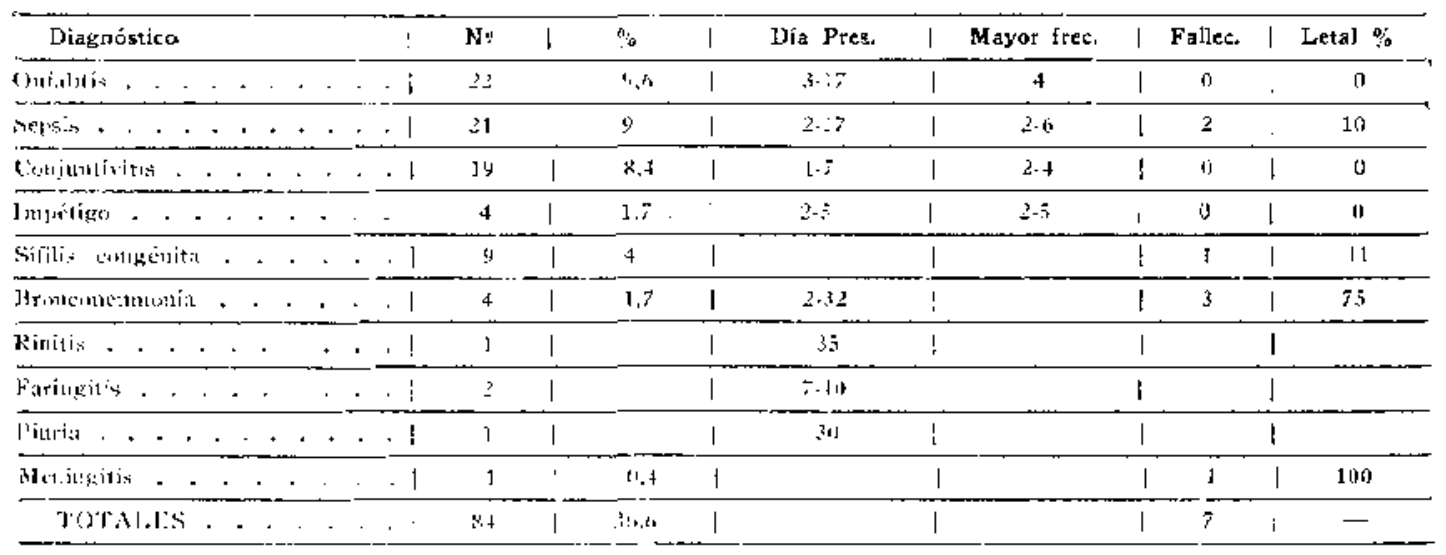

fras y nos orientaría para un estudio más amplio.

Asi, hemos podido comprobar nuestra pobreza en lo que se refiere a personal médico y para-médico, equipo y estadística, condiciones todas posibles de subsanar, por lo menos en sus aspectos fundamentales. $Y$ esta necesidad resulta imperiosa, al ver cómo se hace cada día más abundante la literatura nacional y extranjera sobre este tema y cómo se demuestra en ella el progreso asistencial y la disminución de la mortalidad del prematuro.

Al analizar, ahora, nuestros resultados, queremos insistir en que no pretendemos derivar conclusiones definitivas, sino que simplemente exponerlas, pese a las comparaciones a que las hemos sometido frente a los otros autores.

Se ve que nuestro porcentaje de prematuridad $(6,42 ; ;)$ cae dentro de Ias cifras que se leen en las comunicaciones a nuestro alcance y que la mortalidad global es $20,08 \%$. Esto aparentemente no revestiría suma gravedad. Sin embargo, desglosando este rubro y reduciendo la muestra a los menores de $2.000 \mathrm{gr}$, aparece la proporción alarmante que alcanzan las defunciones $(67,8 \%)$.

La frecuencia y distribución de fallecimientos en orden cronológico y sus causas, coinciden en general con otras estadísticas. Esta misma coincidencia la observamos al comparar la mortalidad de prematuros nacidos de madres sanas $\left(16,4^{*} \%\right.$ con la de aquellos cuyas madres presentaban alguna alteración sistemática $(25,8 \mathrm{c})$. Esto demuestra la importancia del control y tratamiento adecuados de las complicaciones del embarazo.

Es difícil analizar la mortalidad derivada de los distintos tipos de parto ya que muchas veces éste es determinado por complicaciones maternas que exigen intervención inmediata, no sólo para lograr la sobrevida del nin̄o, sino que, frecuentemente, para salvar la vida de la madre. Por lo tanto, las cifras revelan una mortalidad producida por una complicación materna y no por una determinada atención obstetrica.

Considerando en conjunto las causas de prematuridad, llama la atención el porcentaje que alcanzan las causas desconocidas, lo que incide en la necesidad de profundizar su estudio. Es notoria, por otro lado, la gran frecuencia de la toxemia gravídica y el escaso número de causas fetales.

La hemorragia intracraneana aparece como la causa principal de mortalidad en la primera semana y las infecciones en las semanas siguientes.

Al estudiar la asfixia sorprende su me. nor proporción en relación con cifras clásicas, lo que atribuimos simplemente a la falta de una asistencia completa, puesto que hemos logrado pesquisar más casos que los rotulados en las fichas de los niños, gracias a la revisión de las historias de las madres.

Es muy significativa la alta proporción de todos los tipos de infecciones, situación condicionada seguramente por la modalidad de trabajo, es decir, escasez de ropa, de personal y recursos en general, y el hacinamiento tanto de madres como de niños. 


\section{RESUMEN}

Se revisa el materjal de prematuros nacidos en el Hospital Clínico Regional durante el año 1954 y la asistencia brindada a ellos en las Secciones de Puericultura e Incubadora.

Ese año nacieron 3.857 niños vivos en la Maternidad, de Ios cuales 248 fueron prematuros $(6,42 \%)$. Poco más de los dos tercios $(69,86 \%)$ fueron atendidos en las salas de Puericultura y el resto $(30,13 \%)$ en Ia sala Incubadora.

El hacinamiento existente se traduce en Ia frecuencia de las infecciones $(36,68 \%)$.

Aunque la mortalidad global es moderada $(20,08 \%)$, la cifra de fallecidos con peso inferior a $2.000 \mathrm{gr}$. es alarmante $(67,84 \%)$.

La mortalidad en relación con el sexo, la paridad, el embarazo único o múltiple, el estado de salud y control de la madre y la frecuencia y distribución en el tiempo, siguen valores semejantes a los que comunican otros autores.

Al igual que en otras partes, la incidencia de causas desconocidas de prematuridad es elevada $(39,73 \%)$ y, la toxemia gravidica es la más importante dentro de las causas maternas $(32,5 \%)$ entre nosotros.

La asfixia se hizo presente en un $23,1 \%$ con una mortalidad de $47,1 \%$ y nos explicamos su baja frecuencia por la falta de una asistencia integral del prematuro.

\section{SUMMARY}

The Premature Infant born IN The District Hospital of Concepción in 1954.

The case histories of premature infants born in the District Hospital of Concepción in 1954 are reviewed, and the care they received in the Newborn Infants Department and Incubator is detailed.

During this year, 3.857 live-born infants were born in the Maternity, of whom 248 were prematures $(6,42 \%)$. Of these, two thirds $(69,86 \%)$ were attended in the Newborn Infant Department and the others $(30,13 \%)$ in the Incubator.

Due to overcrowding, infections were numerous $(36,68 \%)$. An alarming number of infants under $2.000 \mathrm{gr}$. died $(67,84 \%)$, although general mortality rate was moderate $(20,08 \%)$.
We found that our mortality offers no difference in relation to sex, number of deliveries, single or multiple pregnancy, health of the mothers and pre-natal control, frequency and time, with the values given by other authors.

The incidence of prematurity due to unknown causes is high $(39,73 \%)$ and eclampsia is the most important maternal cause $(32,5 \%)$.

Asphyxia was present in $23,1 \%$, with a fatality rate of $47,1 \%$; this small frequency is explained by the lack of adequate care of the premature infant.

\section{BIBLIOGRAFIA}

1. -NILEN, M, y LTEV1NE, A. -- "Rejmonse of small Tremature inciants to restriction ai Supplementary Oxi(ve1)". Am. J. Jjis, Claikl. $89: 396,1955$

2.-AL.I, 1, - "Tlie ear'y nroutlosis for prenteluted with

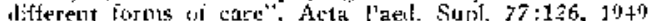

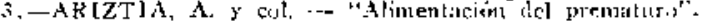
Jev. Chilen: de ped $25: 305,1954$.

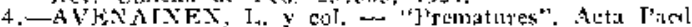
(1):171 19 is?

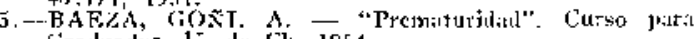
Graduntus. 1. de ti. 1954 .

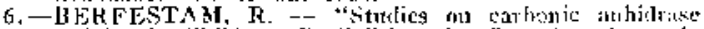
activity in Childien. Posibilities oi iniluencing the earls. anhiolr. activity in the hived of prenatere infants". Sita T2:

7,-BERFFSTAM, R. - "Sturlies en cirlt, anhitlr. iu 11,

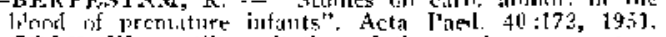

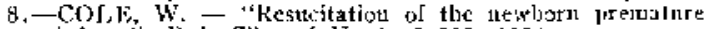
infants. ]'tl, Clin, of N, A. 8:527, 1954,

9.--COOKF, ]2. - "Cate of premature infitat". 1'eel. (lin. of $N$ A. \& : fif9. 1954.

10. -CRALFORD, Hi, i. "Newer cincerts of premature care". Fed, thim oi N. A. 8:659, 1954.

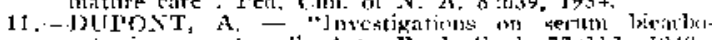
nate in fornatures". Acla Paul. Sunl. $7: 11,3$ J Jy.14.

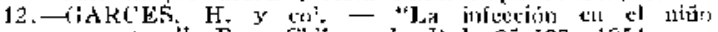
prematneos, Rey, Chitectia le T'el, 25:I09, 1954.

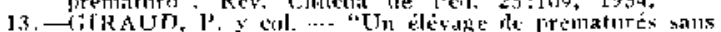

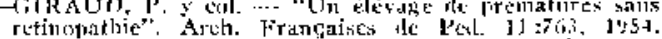

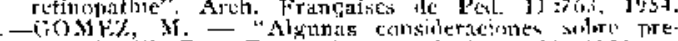

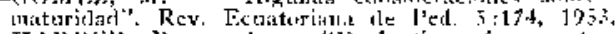

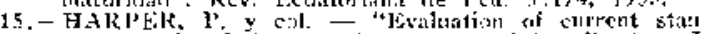

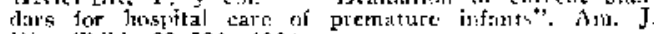
1)is. (Yhilol. 88:523, $19: 4$.

ii.--IIILI, J. S. - "l'ritctice of fluicl therafty is I'erlia-

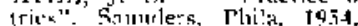

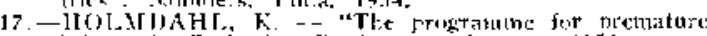

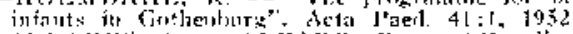

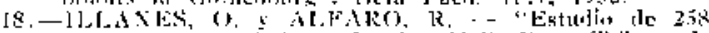

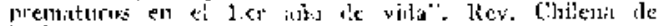

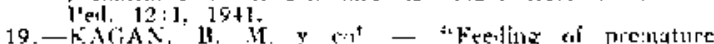

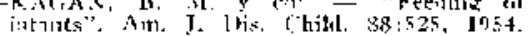

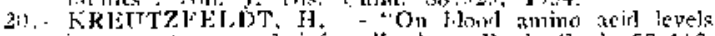

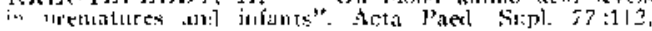
1949 .

2I.-IABATE, 5. y TUI'KSOX. W, ... "Prevention of

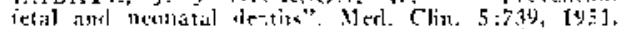

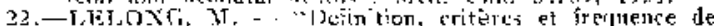
la licemattirite". Stmaille des Hôp. $75: 29.17,1951$.

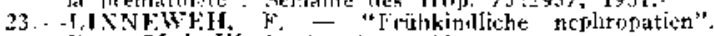

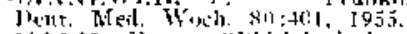

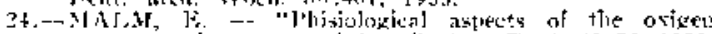

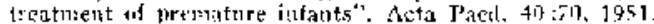

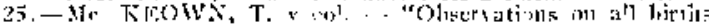

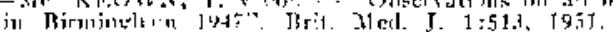

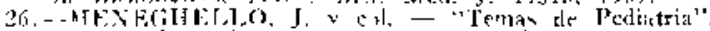

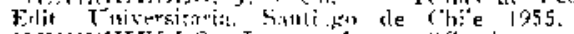

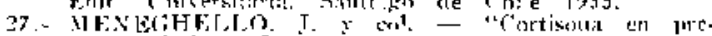

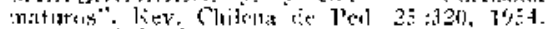

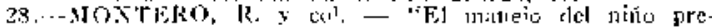

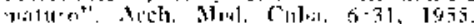

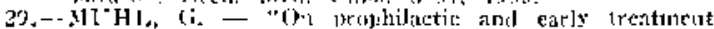
of iniecilons in newikertse especially Prematures". $A$ ". tit F'iect. $38: 201,10 \$ 9$.

3) - ONTEGA, K. y REPETTO, G, - "Viknación anti 


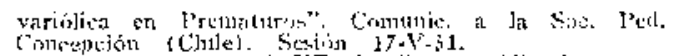

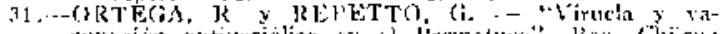

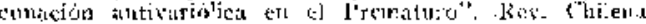

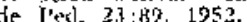

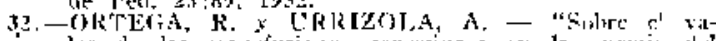

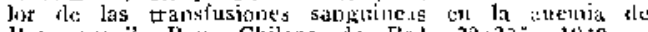
l'rematuro". Rev. Chileos de Perl. 2ik:23. 1949.

33. - IRTEGA, R. - "El prematino en las clinicits $y$ ed

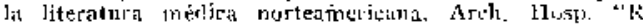
tiel kis;". 12:44, 19.1t.

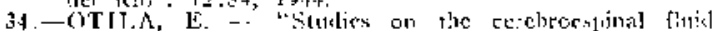

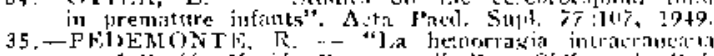

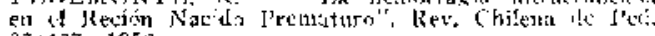
$23: 427,105 \%$.

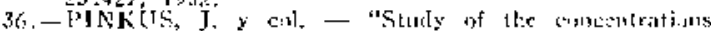

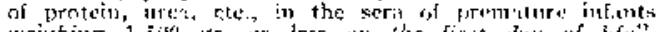

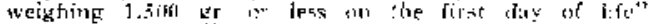

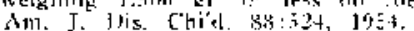

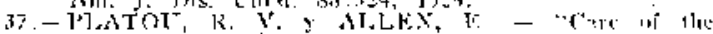

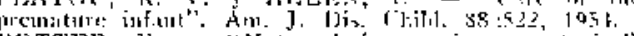

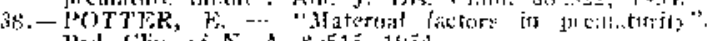
]

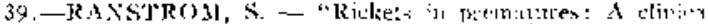

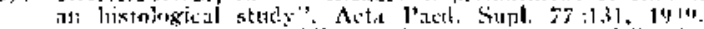

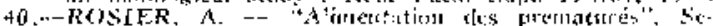
maiue les Hôp. $75: 2958$, 1049

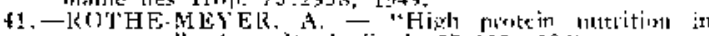
premature", Asia l'uerl. Sinpl. 7t:109, $194 \%$.

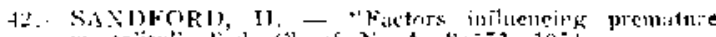

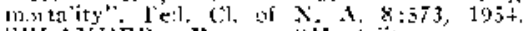

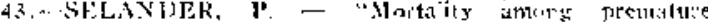
inituts. Acta Tael. 38:5\%, 1949.

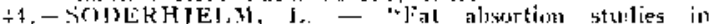

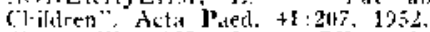

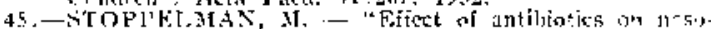
pharingral flirra in potemature infant;". Art. J. Dis.

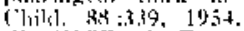

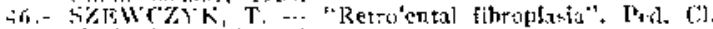
uा $x$, sing 1954

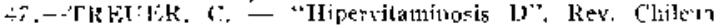
le pel. it: 170, ? 953 .

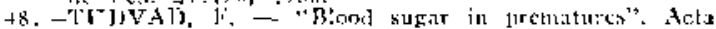

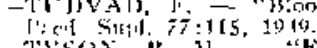

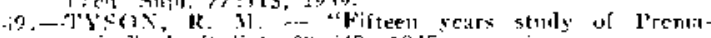

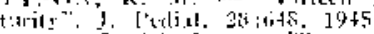

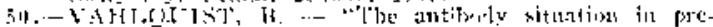

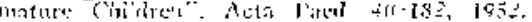

51. - rlavis

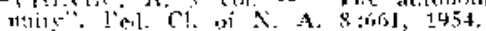

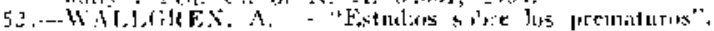

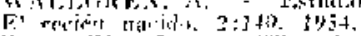

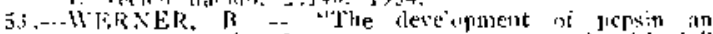

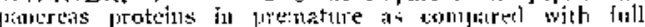

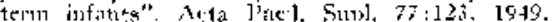

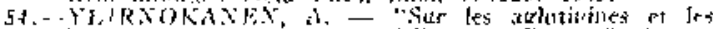

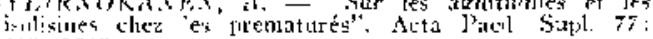
$11+19+19$ 